\title{
English Translation of China's Internet Buzzwords from the Perspective of Skopos Theory
}

\author{
Hu Tianchen, Zheng Tingyun* \\ North China Electric Power University \\ Beijing, China
}

\begin{abstract}
With the development of network and globalization, China's Internet buzzwords gradually become popular. Accordingly, accurate and concise translation of these words has become increasingly important for the spread of these words and the culture they contain. This paper analyzes the features of China's internet buzzwords from the perspective of Skopos Theory and summarizes translation methods. This paper aims to provide some reference and guidance for the translation of China's Internet Buzzwords.
\end{abstract}

Keywords-translation; buzzwords; skopos theory; crossculture communications

\section{INTRODUCTION}

Science and technology are developing rapidly these years so it is not surprisingly Internet plays an increasingly important role in people's life. Browsing social media has become an important channel to gain information. With the popularity of the Internet, the whole world has been connected into the Internet system, which has become a "global village". Through the Internet, people can instantly obtain information from all corners of the globe. Compared with the past, it is faster and more convenient.

With the increasing influence of China, more and more people begin to learn Chinese, and they are eager to have an in-depth understanding of Chinese culture and to know about some latest Internet buzzwords. So it is really natural to follow the trend to introduce some trending topics and buzzwords among the Chinese netizens to the foreign learners. For buzzwords, there are many kinds of translations of the buzzwords on the Internet, but sometimes, the translation quality is difficult to guarantee due to the rich cultural connotation and environment behind those words. Therefore, to translate Chinese buzzwords accurately and exactly for foreign readers to have a better understanding, we need to deal with these difficulties. And in this way, Skopos Theory is suitable acting as our translation instructive theory.

\section{SKOPOS THEORY}

Skopos theory was put forward by Hans Vermeer, a German linguist and translation scholar. Skopos theory has three rules: the Skopos rule, the Coherence rule and the Fidelity rule, in which the Fidelity rule should be subordinate to the Coherence rule and they both are subordinate to the Skopos rule.

The Skopos rule requires translators to focus on the aim or the purpose of the translation which will involves the situation and target readers. The Coherence rule emphasizes target text's readability by target readers in the particular situation. The Fidelity rule focuses on the corresponding relationship between the source text and the target text. They can be summarized to that "translation is the cross-cultural communication activities that have clear purpose and intention, based on the original text."(ZHENG 1157)

\section{THE FEATURES OF CHINESE ONLINE BUZZWORDS}

Chinese online buzzwords have their unique features related with the Chinese culture and the features of the cyberspace. China possesses a splendid culture which influences generations of Chinese people. Naturally, Chinese netizen will exhibit this cultural influence. On the other hand, to a majority of people, Cyberspace has become an indispensable part of their daily life, which undoubtedly decides the popularity of those words.

\section{A. Cultural Connotation}

Some online buzzwords have been broadly used in people daily lives for a long time before the appearance of Network. Then, as the network gradually step into people's daily live, they became popular on the Internet accordingly. Many of these words have come into being in the ancient China and are connected with the customs and beliefs of Chinese people. For example, the buzzword Koi Fish (Jin Li) becomes popular because people believed that it can bring good luck and this connotation has existed since Spring and Autumn period (770BC-403BC). Nevertheless, not all these words are connected with Chinese culture. Some of them are related with western cultures, such as Mercury Retrograde (Shui Ni) which origins from astrology. 


\section{B. Entertainment Functions}

Many people regard surfing Internet as an entertainment. Thus, online buzzwords would also show their entertainment functions. The functions can be divided into two types. Firstly, people want use some words to praise others in an interesting way, such as the word 666 which means doing well. Secondly, some words can be used to ridicule or satirize people in a euphemistic way, like Lemon Essence(Ning Meng Jing) which refers to the people who are always jealous of the achievements or happy life of other people. These words are always amusing and have become an important component of people's daily communication.

\section{Concise Style}

Another reason for buzzwords' popularity is their concise style. Understanding and remembering are the foundation of usage. Consequently, people are inclined to use those concise words rather than the complex and long words or sentence to both save time and reduce communication barriers. Generally speaking, Chinese online buzzwords consists of two characters, such as Official Announcement (Guan Xuan) and Extend Buddy(Kuo Lie). And the longest ones will not exceed 5 characters. What's more, their meanings are expressed clearly by the characters or are not difficult to guess with a little imagination or background knowledge. For example, the meaning of Lemon Essence origins from the sour taste of lemon, which is similar to the bitter feeling of jealousy.

\section{Timeliness}

Cyberspace changes quickly, so does people's interest. "Buzzwords always originate from a particular event or phenomenon. Enhance, they will gradually disappear with the attention on the event or phenomenon fading."(HE 10) The word Double Eleven(Shuang Shi yi) is a typical case. Double eleven is the shopping carnival on November 11th, originally held by Tmall, a famous Chinese e-commerce platform. On that day, the online retailers will promote just like the Black Friday. So consumers will be excited and also talk about it shortly before and after the day. However, people will stop talk about it when their passion has faded and won't pay attention to it until the day of next year. What's more, not all words will be reminded of, a majority of them will not be popular again without another stimulus.

\section{ENGLISH TRANSLATION ON CHINESE ONLINE BUZZWORDS}

When we are translating, translators must combine the context and culture of the target language to select different translation strategies to translate. Here are some examples selected from our practices. See Table 1.
TABLE I. TRANSLATION METHODS AND EXAMPLES

\begin{tabular}{|l|l|}
\hline Methods & Examples \\
\hline Literal Translation & $\begin{array}{l}\text { official announcement; } \\
\text { Double Eleven }\end{array}$ \\
\hline Liberal Translation & $\begin{array}{l}\text { playboy ; } \\
\text { break one's promise }\end{array}$ \\
\hline Amplification & Pan Ta \\
\hline Omission & $\begin{array}{l}\text { argumentative people ; } \\
\text { debut as central person }\end{array}$ \\
\hline Back-translation & $\begin{array}{l}\text { Hardcore; } \\
\text { Mercury retrograde }\end{array}$ \\
\hline Creative Translation & wo-man (women with a tough heart) \\
\hline
\end{tabular}

\section{A. Literal Translation}

When the form and content of the original text are exactly equivalent to that of the target language, according to the Coherence rule, we can just use literal translation, which won't affect readability.

\section{Eg.1 “Guan Xuan”-official announcement}

"Guan Xuan" is a word became popular in Oct.16th, 2018 because Chinese actor Feng Shaofeng and actress Zhao Liying used "Guan Xuan" to officially announced their marriage. We translated into official announcement, and they are equal in both meaning and function. Both of the words means people involved in an event announce their decisions or plans officially and publicly.

We can also use literal translation, when the word represents some things such as an activity or a customs unique in China. Under this circumstance, we will also give some explains about the word's resources and meaning.

\section{Eg.2 "Shuang Shi Yi”- Double Eleven}

Double Eleven Shopping Carnival is a network promotion day, on every year' $s$ Nov. 11th. November 11th became a fixed date for Tmall to hold large-scale promotional activities because merchants can gain massive profits from this activity. Here, Double Eleven is a unique activity in China, so an equivalent translation could not be found. Thus, we also use literal translation with further explains.

\section{B. Liberal Translation}

According to the Fidelity rule, when meaning and form cannot be taken into account in the process of translation, we should pay more attention to the equivalence of meaning between the original text and the translated text, and choose the equivalence of meaning instead of the equivalence of language form.

\section{Eg.3 “Da Zhu Ti Zi”-playboy}

This word "Da Zhu Ti Zi" originally refers trotter, while here we used to say the man who is not loyal or honest. So when we translate into English, we cannot directly translate it word by word. Instead, we use the word playboy, which has similar meaning in English, and foreign reader will easily understand.

\section{Eg.4 “Zhen Xiang”-break one's promise}

The original meaning of "Zhen Xiang" is that the food smells so delicious. However, this is not what Chinese 
netizens want to express. It has a background story that an urban boy went to a rural family for the first time. When he arrived at there, he said he would never eat food there. But later, he ate the food in an enjoyable way, saying so delicious. So we translate it into break one's promise, the meaning really popular on the Internet.

\section{Amplification}

Chinese and English are not completely equivalent, so, it is inevitable that there will be cultural and background differences. Fidelity rule suggests us to add more explains in the translation to guarantee the rounder correspondence between source and target text. What's more, some words have different meaning in different contexts.

\section{EG.5 "Pan Ta"}

This word comes from the crosstalk "Collectables" performed by Meng Hetang and his partner Zhou Jiuliang two Chinese crosstalk performers from Deyun Crosstalk Association. It originally means to polish which means turning around something in one hand, in order to make its surface smoother. The second meaning is to teach the people you hate a lesson. The third meaning is to hit on the people you like. The last meaning is to stroke the lovely things unconsciously. So when we translated this word, we need to make additional explains accordingly.

\section{Omission}

According to the Coherence rule, when some words that do not conform to the thinking habits, language habits and expressions of the target language, they should be deleted to avoid misunderstandings and confusions.

\section{Eg.6"Gang Jing”-argumentative people}

"Jing" originally means hobgoblin, but here it uses personification, so it refers to people in "Gang Jing". Consequently, when we translate this word, "Jing" or hobgoblin is omitted and we just remains its meaning of people.

\section{Eg.7 "C Wei"- debut as central person}

In this word, "Wei" represents position in Chinese. However, this word is widely used in Entertainment Industry to a singer or artist who has strong ability in the group and always stands at the central palce with relatively more shots and being remembered quickly. As a result, while translating, we omitted position, but central person.

\section{E. Back-translation}

In cultural exchanges, Chinese also absorbs a lot of vocabulary from English. Therefore, according to the Fidelity rule, we could use the original English in translation to ensure correspondence.

\section{Eg.8 "Ying He"-hardcore}

"Ying He" is actually the Chinese translation of hardcore, so it originally comes from English. Thus, when we translate it back to English, we don't need to find another word but its original words, which is very important to guarantee accuracy and avoid confusion.

\section{Eg.9 "Shui Ni"-Mercury retrograde}

"Shui $N i$ " is also a foreign word. It is a kind of visual illusion that the moving direction of Mercury is retrograde. However, this phenomenon has also been observed by ancient the Chinese, but it "Shui Ni" here has its special meaning in divination which comes from the west. So, during translation, we translate it back into English as Mercury retrograde.

\section{F. Creative Translation}

When other translation methods can not express the meaning well, we may break the traditional ways of translation and consider creative translation. heart)

Eg.10 "Nv Han Zi"-wo-man (women with a tough

For the 2013 buzzword "Nv Han $Z i "$, it refers to those women who think or are considered by the public to be similar to men in character, words and deeds. "On the Internet, there are some translation versions like manly woman, female man, etc. Although the first and the second translation has similar meanings, it is easily to be misunderstood. If we use the innovative translation "wo-man (women with a tough heart)" it can better express the original meaning." (Wang 283) In form, it is similar to woman; while in sound, it pronounces differently. As a result, when other methods are not as good as we think, we can think of creative translation.

\section{CONCLUSION}

As an important way for foreigners to learn Chinese language and culture, Internet buzzwords are of great significance in cross-cultural communication. It's also important to translate buzzword well. Because of the features of China's internet buzzwords, before translation, translators are required to delve into the words' connotation and background information. Then, under the guidance of the Skopos theory, translators could comprehensively apply translation skills to guarantee the readability and correspondence of the translation in order to give a rounder explains for the foreign readers to understand the words.

\section{REFERENCES}

[1] ZHENG Tiantian, "An Analysis of Film Subtitle Translation from Perspective of Skopos Theory," Proceedings of Conference on Creative Education (CCE2012). China, pp. 1157-1158, 2012. (In Chinese)

[2] HE Minggang, "Features of Internet Buzzwords and Their ChineseEnglish Translation Methods," Journal of Luoyang Institute of Science and Technology (Social Science). China, vol. 30, pp. 8-13, 2015. (In Chinese)

[3] Wang Xinping, "A preliminary study on the English translation strategies of Internet buzzwords under the influence of Skopos Theory," Foreign Language Education and Translation Development Innovation Research. China, vol. 8, pp. 281-283, 2019. (In Chinese) 